\title{
Risk Factors for Tigecycline-Associated Hypofibrinogenemia
}

This article was published in the following Dove Press journal:

Therapeutics and Clinical Risk Management

\author{
Jia Liu' \\ Yingying Yan $^{2}$ \\ Fan Zhang $\mathbb{D}^{\prime}$ \\ 'Department of Geriatrics, Peking \\ University Third Hospital, Beijing, \\ People's Republic of China; ${ }^{2}$ Department \\ of Pharmacy, Peking University Third \\ Hospital, Beijing, People's Republic of \\ China
}

Background: With the widespread use of tigecycline, especially in elderly people infected with multidrug-resistant bacteria, the associated coagulation disorders are attracting the attention of clinicians. The risk factors of tigecycline-associated hypofibrinogenemia are still controversial.

Purpose: The aims of our study were to explore the related factors of hypofibrinogenemia caused by tigecycline, and to establish the risk assessment criteria for tigecycline-associated hypofibrinogenemia.

Patients and Methods: This was an observational retrospective cohort study of patients treated for at least 3 days with tigecycline. Hypofibrinogenemia was defined as plasma fibrinogen $<2.0 \mathrm{~g} / \mathrm{L}$. Risk factors were determined using logistic regression analysis, and the risk assessment criteria were identified by using receiver operating characteristic curves. Results: In total, 148 patients were included in the analysis, mean age was $77.09 \pm 15.11$ years old. Ninety patients who developed hypofibrinogenemia during tigecycline treatment with mean plasma fibrinogen of $1.42 \mathrm{~g} / \mathrm{L}$ were included in the hypofibrinogenemia group, the other 58 patients with mean plasma fibrinogen of $2.68 \mathrm{~g} / \mathrm{L}$ were included in the normal group. In the multivariate analysis, age $(p=0.035)$, tigecycline treatment duration $(p=$ $0.044)$, and baseline fibrinogen level $(p=0.002)$ were independently associated with hypofibrinogenemia. An age of $\geq 82$ years, $\geq 9$ days of medication, and a baseline fibrinogen level of $\leq 3.5 \mathrm{~g} / \mathrm{L}$ were selected for predicting hypofibrinogenemia. Hypofibrinogenemia was independently associated with bleeding (OR 8.96, 95\% CI [1.132-70.946], $p=0.038$ ).

Conclusion: Hypofibrinogenemia is a common adverse effect of tigecycline in our study. Elderly patients are more prone to developing hypofibrinogenemia after the administration of tigecycline. It is independently associated with bleeding but not death. The risk assessment criteria can help in the identification of patients with high risk of hypofibrinogenemia.

Keywords: tigecycline, hypofibrinogenemia, coagulation disorders, risk factors

\section{Introduction}

Tigecycline is the first antibiotic in the glycylcycline class and has broad-spectrum activity, especially against multidrug-resistant bacteria, such as methicillin-resistant Staphylococcus aureus (MRSA), vancomycin-resistant Enterococcus (VRE) strains, and multidrug-resistant Acinetobacter baumanii (MDRAB). ${ }^{1,2}$ Because it has high levels of antibacterial activity and tolerability, it is widely used for the treatment of community-acquired pneumonia, complicated skin infections, and intra-abdominal infections. ${ }^{3,4}$ Advanced age and chronic diseases are risk factors related to multidrugresistant bacteria infections. ${ }^{5}$ Because of the efficacy and safety, tigecycline is often used in elderly people infected with multidrug-resistant bacteria in clinical practice. ${ }^{6}$
Correspondence: Fan Zhang

Department of Geriatrics, Peking

University Third Hospital, 49 North

Garden Road, Beijing, I00191, People's

Republic of China

Tel +86-0l0-82265685

Email zhangfan_puh3@yeah.net 
The most common adverse drug reactions of tigecycline include nausea, vomiting and diarrhea. ${ }^{7}$ However, clinicians often overlook a very rare adverse effect, coagulopathy, which occurs in only less than $2 \%$ of all patients in premarketing clinical trials. ${ }^{8}$ With the widespread use of tigecycline, the associated coagulation disorders are attracting the attention of clinicians. In addition to the prolongation of the prothrombin time (PT) and activated partial thromboplastin time (aPTT), hypofibrinogenemia has also been reported. ${ }^{9-11}$ We should pay more attention to adverse effects in the elderly patients in clinical practice. Risk factors for tigecyclineassociated hypofibrinogenemia were unclear. According to few clinical studies, the relevant factors may include renal insufficiency, the treatment dose and course, patient sex and plasma fibrinogen level before administration, but the conclusions are controversial. ${ }^{12-15}$ Moreover, previous studies have only described the relevant risk factors without effective risk assessment criteria, which can aid the selection of antibiotic drugs and reduce the occurrence of adverse reactions.

In this article, we conducted a retrospective observational study to explore the related factors of hypofibrinogenemia caused by tigecycline and to establish the risk assessment criteria for tigecycline-associated hypofibrinogenemia.

\section{Materials and Methods Study Population}

A retrospective, single-center, observational cohort study was performed at Peking University Third Hospital. Patient data were retrieved from the Pharmacy Department database. The Medical Science Research Ethics Committee of the Peking University Third Hospital approved this study. We identified 203 patients who were treated with tigecycline in 2019. Then, we excluded 55 patients because they met one of the following four criteria: (1) patients who were treated with tigecycline for fewer than 3 days; (2) patients who were treated with tigecycline without coagulation status monitoring; (3) patients with a fibrinogen level less than $2.0 \mathrm{~g} / \mathrm{L}$ before the administration of tigecycline, and (4) patients who were diagnosed with disseminated intravascular coagulation (DIC). Finally, 148 patients were included in the analysis.

\section{Study Setting}

According to the case report form, we collected clinical information from the electronic medical records system. The data we collected included demographic and medical history information (eg, age, sex, height, weight, and chronic diseases), the results of laboratory tests performed before, during and 1 week after the treatment of tigecycline (eg, leukocyte count, hemoglobin level, platelet count, transaminase level, albumin level, creatinine level, urea nitrogen level, prothrombin time, activated partial thromboplastin time, thrombin time, fibrinogen level, procalcitonin level), the site of infection, the tigecycline dosage, the duration of tigecycline treatment, concomitant drugs (anticoagulants, antiplatelet drugs, cefoperazone/sulbactam), and clinical outcomes (eg, bleeding, death). All laboratory tests were conducted in the same laboratory. Serum fibrinogen level was measured with HemosIL Fibrinogen-C reagents (Instrumentation Laboratory) using von Clauss assay. The laboratory reference range for fibrinogen was $2.0-4.4 \mathrm{~g} / \mathrm{L}$. Hypofibrinogenemia was defined as plasma fibrinogen $<2.0 \mathrm{~g} / \mathrm{L}$. The primary outcome was the occurrence of hypofibrinogenemia after tigecycline treatment, and the secondary outcome was the incidence of adverse events (eg, bleeding, death).

\section{Statistical Analysis}

Statistical analysis was performed with SPSS statistics 19.0. The continuous variables are presented as means \pm standard deviations (SDs) (normally distributed) or medians and interquartile ranges (non-normally distributed). We evaluated these variables by the $t$ test or MannWhitney $U$-test. The categorical variables are presented as percentages, and Fisher's exact test or the Chi-square test was used to evaluate these variables. Multivariable logistic regression models were used in association analyses. $P<0.05$ was regarded as statistically significant. The goodness of fit was assessed with the HosmerLemeshow test, and the discrimination threshold was determined by receiver operating characteristic (ROC) curve analysis. The area under the curves (AUCs) and the corresponding 95\% confidence intervals (CI 95\%) were shown.

\section{Results}

The mean age of the 148 patients was $77.09 \pm 15.11$ years old. $88.5 \%$ of the patients were over 60 years old. We included 108 males and 40 females. Among these patients, 141 were diagnosed with pneumonia, 17 were diagnosed with bloodstream infection, 9 were diagnosed with intraabdominal infection, and 2 were diagnosed with intracranial infection. Tigecycline was mainly used for the treatment of multidrug-resistant bacterial infections, such 
as Acinetobacter baumannii, Klebsiella pneumoniae, Escherichia coli, and MRSA. Among these patients, 133 received normal-dose treatment (loading dose $100 \mathrm{mg}$, maintenance dose $50 \mathrm{mg} \mathrm{Q} 12 \mathrm{H}$ ), and 15 patients received high-dose treatment (loading dose $100 \mathrm{mg}$ or $200 \mathrm{mg}$, maintenance dose $100 \mathrm{mg} \mathrm{Q} 12 \mathrm{~h}$ ). The average duration of tigecycline treatment was $12.01 \pm 5.73$ days. The effect of tigecycline on blood coagulation manifested as a significant decrease in the plasma fibrinogen level, and a significant increase in aPTT, PT and thrombin time (TT). The fibrinogen level decreased from $3.76 \pm 1.12 \mathrm{~g} / \mathrm{L}$ to 1.91 $\pm 0.78 \mathrm{~g} / \mathrm{L}$ and recovered to $2.66 \pm 0.54 \mathrm{~g} / \mathrm{L}$ after drug withdrawal $(p<0.001)$. The activated partial thromboplastin time increased from $33.5 \pm 5.6 \mathrm{~s}$ to $41.6 \pm 10.7 \mathrm{~s}$ and recovered to $38.1 \pm 8.0 \mathrm{~s}$ after drug withdrawal $(p<0.001)$. The prothrombin time increased from $13.1 \pm 2.3 \mathrm{~s}$ to $15.3 \pm 4.9$ $\mathrm{s}$ and returned to $13.1 \pm 1.88 \mathrm{~s}$ after drug withdrawal $(p<$ $0.001)$. The thrombin time increased from $15.37 \pm 3.91 \mathrm{~s}$ to $19.47 \pm 6.28 \mathrm{~s}$ and returned to $16.56 \pm 2.18 \mathrm{~s}$ after drug withdrawal $(p<0.001)$. After treatment with tigecycline, the fibrinogen level decreased by an average of $49.2 \%$, PT, aPTT and TT increased by an average of $16.8 \%, 19.5 \%$ and $26.7 \%$, respectively. We found no significant changes in the D-dimer levels in response to the treatment.

A total of 142 patients $(95.9 \%)$ in the study experienced a decrease in the fibrinogen level during tigecycline treatment, and 90 patients $(60.8 \%)$ developed hypofibrinogenemia. The patients who developed hypofibrinogenemia were included in the hypofibrinogenemia group. Moreover, the other 58 patients who had fibrinogen $>2.0$ $\mathrm{g} / \mathrm{L}$ during tigecycline treatment were included in the normal group. The clinical characteristics of the two groups are shown in Table 1. In the univariate analysis, the ages of the hypofibrinogenemia group and the normal group were $79.06 \pm 14.41$ and $72.97 \pm 18.08$ years old $(p=$ $0.025)$, and the tigecycline treatment duration were 12.86 \pm 6.04 and $10.69 \pm 4.97$ days $(p=0.024)$. There were no significant differences between the two groups in sex, body mass index or medication dosage. There were no differences between the two groups in the site of infection or the presence of underlying diseases (hepatobiliary system diseases, chronic renal insufficiency, and malignant tumors). In the hypofibrinogenemia group, 22 (24.4\%) patients were treated with cefoperazone/sulbactam, 13 (14.4\%) patients were treated with anticoagulant drugs, and $16(17.8 \%)$ patients were treated with antiplatelet drugs. There were no differences between the two groups in the combination therapy with anticoagulant, antiplatelet drugs or cefoperazone/sulbactam. There were differences between the two groups in the baseline hemoglobin, total protein, urea nitrogen and fibrinogen levels before medication. In the hypofibrinogenemia group and the normal group, the baseline fibrinogen levels were $3.46 \pm 1.01 \mathrm{~g} / \mathrm{L}$ and $4.21 \pm 1.12 \mathrm{~g} / \mathrm{L}$, and decreased to $1.42 \pm 0.38 \mathrm{~g} / \mathrm{L}$ and $2.68 \pm 0.57 \mathrm{~g} / \mathrm{L}$ during treatment, respectively. In the hypofibrinogenemia group, the fibrinogen level of 12 patients $(13.3 \%)$ decreased to less than $1 \mathrm{~g} / \mathrm{L}$. The hypofibrinogenemia group had a $56.2 \%$ fibrinogen decrease and the normal group had a 33.8\% fibrinogen decrease $(p<0.001)$.

The following variables that were significant at $p<$ 0.05 in the univariate analyses and risk factors in previous studies were included in the multivariate analysis: age, sex, treatment duration, hemoglobin level, creatinine level, urea nitrogen level, basal fibrinogen, total protein. In the multivariate analysis (stepwise approach), age ( $p=$ $0.035)$, tigecycline treatment duration $(p=0.044)$, and baseline fibrinogen level ( $p=0.002)$ were independently associated with hypofibrinogenemia (Table 2).

The ROC curve was used to evaluate the predictive ability of age, tigecycline treatment duration and the baseline fibrinogen level for tigecycline-related hypofibrinogenemia. Area under curve (AUC) of age was 0.621 (95\% CI: $0.530-0.712, p=0.013$ ), tigecycline treatment duration was 0.596 (95\% CI: $0.501-0.691, p=0.049)$, and baseline fibrinogen level was 0.713 (95\% CI: 0.630-0.796, $p<0.001)$. We selected the cutoff point with the largest Youden index. An age of $\geq 82$ years, $\geq 9$ days of medication, and a fibrinogen level of $\leq 3.5 \mathrm{~g} / \mathrm{L}$ were selected as the cutoff points for predicting hypofibrinogenemia during tigecycline treatment (Table 3).

Among the 148 patients who received tigecycline, 13 $(8.8 \%)$ developed bleeding. The main bleeding sites were the digestive tract $(84.6 \%)$, skin and mucosa (15.4\%). There were differences between the two groups in the complication of bleeding $(p=0.014)$. In the hypofibrinogenemia group, 12 developed bleeding. Five patients received only fresh frozen plasma infusion, two patients discontinued tigecycline without any other treatment for hypofibrinogenemia, two patients discontinued tigecycline and received plasma infusion, one patient received prothrombin complex, fibrinogen, and transfusion of plasma, one patient received vitamin $\mathrm{K}$ and transfusion of plasma. Their fibrinogen levels were increased after the above treatment. Disease progressed rapidly in one patient, and died without treatment for hypofibrinogenemia. The hospital mortality rate of the 
Table I Univariate Analysis of Risk Factors for Tigecycline-Associated Hypofibrinogenemia

\begin{tabular}{|c|c|c|c|}
\hline & Normal Group & Hypofibrinogenemia Group & P-value \\
\hline$N$ & 58 & 90 & \\
\hline Age (years) & $72.97 \pm 18.08$ & $79.06 \pm|4.4|$ & $0.025^{*}$ \\
\hline Sex (male, $n(\%))$ & $46(79.3)$ & $62(68.9)$ & 0.163 \\
\hline Body mass index $\left(\mathrm{kg} / \mathrm{m}^{2}\right)$ & $22.74 \pm 4.5$ I & $22.4 I \pm 4.44$ & 0.706 \\
\hline Dose $(200 \mathrm{mg} / \mathrm{d}, \mathrm{n}(\%))$ & $4(6.9)$ & II (I2.4) & 0.429 \\
\hline Treatment duration (days) & $10.69 \pm 4.97$ & $12.86 \pm 6.04$ & $0.024^{*}$ \\
\hline \multicolumn{4}{|l|}{ Site of infection } \\
\hline Pneumonia (\%) & $56(98.2)$ & $85(96.6)$ & 0.940 \\
\hline Urinary tract infection (\%) & $9(15.8)$ & $16(18.2)$ & 0.710 \\
\hline Bloodstream infection (\%) & $8(14)$ & $9(10.2)$ & 0.486 \\
\hline Intra-abdominal infection (\%) & $4(7.0)$ & $5(5.7)$ & 0.745 \\
\hline Intracranial infection (\%) & $\mathrm{I}(\mathrm{I} .8)$ & $\mathrm{I}(\mathrm{I} . \mathrm{I})$ & 0.755 \\
\hline \multicolumn{4}{|l|}{ Underlying diseases } \\
\hline Chronic renal insufficiency (\%) & $8(14)$ & $18(20.5)$ & 0.325 \\
\hline Hepatobiliary system diseases (\%) & $2(3.4)$ & $9(10)$ & 0.245 \\
\hline Malignant tumors (\%) & $10(17.9)$ & $21(23.9)$ & 0.393 \\
\hline \multicolumn{4}{|c|}{ Laboratory tests before tigecycline treatment } \\
\hline WBC $\left(\times 10^{9} / \mathrm{L}\right)$ & $10.08 \pm 3.83$ & $11.34 \pm 7.90$ & 0.198 \\
\hline HGB $(g / L)$ & $104.05 \pm 21.45$ & $95.78 \pm 20.70$ & $0.021^{*}$ \\
\hline $\operatorname{PLT}\left(\times 10^{9} / \mathrm{L}\right)$ & $195.93 \pm 99.52$ & $182.29 \pm 95.43$ & 0.405 \\
\hline ALT (U/L) & $30.4 I \pm 22.16$ & $31.06 \pm 30.51$ & 0.890 \\
\hline AST (U/L) & $34.38 \pm 22.32$ & $31.84 \pm 22.06$ & 0.478 \\
\hline TBil $(\mu \mathrm{mol} / \mathrm{L})$ & $14.97 \pm 9.76$ & $20.53 \pm 39.14$ & 0.291 \\
\hline ALP (U/L) & $1 \mathrm{I} 2.53 \pm 86.47$ & $93.22 \pm 46.35$ & 0.126 \\
\hline $\mathrm{TP}(\mathrm{g} / \mathrm{L})$ & $65.14 \pm 7.82$ & $61.60 \pm 8.86$ & $0.024^{*}$ \\
\hline ALB $(g / L)$ & $31.07 \pm 3.27$ & $30.50 \pm 4.78$ & 0.407 \\
\hline GGT (U/L) & $83.46 \pm 151.95$ & $54.75 \pm 63.19$ & 0.184 \\
\hline BUN (mmol/L) & $10.74 \pm 6.68$ & $14.11 \pm 10.94$ & $0.022^{*}$ \\
\hline $\mathrm{Cr}(\mu \mathrm{mol} / \mathrm{L})$ & $109.38 \pm 99.15$ & $129.06 \pm 118.89$ & 0.298 \\
\hline eGFR $\left(\mathrm{mL} / \mathrm{min} / \mathrm{I} .73 \mathrm{~m}^{2}\right)$ & $67.76 \pm 33.78$ & $65.94 \pm 32.63$ & 0.782 \\
\hline PT (s) & $12.73 \pm 1.82$ & $13.37 \pm 2.55$ & 0.097 \\
\hline $\mathrm{Fib}(\mathrm{g} / \mathrm{L})$ & $4.21 \pm 1.12$ & $3.46 \pm 1.01$ & $0.000 *$ \\
\hline aPTT (s) & $33.24 \pm 5.01$ & $33.61 \pm 6.02$ & 0.695 \\
\hline D-dimer $(\mu g / d l)$ & $1.27 \pm 1.47$ & $1.61 \pm 2.12$ & 0.324 \\
\hline PCT (ng/mL) & $1.34 \pm 5.25$ & $3.95 \pm 17.33$ & 0.335 \\
\hline \multicolumn{4}{|c|}{ Concomitant drugs associated with, n (\%) } \\
\hline Anticoagulant drugs (\%) & $12(20.7)$ & $13(14.4)$ & 0.322 \\
\hline Antiplatelet drugs (\%) & $5(8.6)$ & $16(17.8)$ & 0.119 \\
\hline CPZ/SAM (\%) & $12(20.7)$ & $22(24.4)$ & 0.596 \\
\hline \multicolumn{4}{|l|}{ Clinical outcomes } \\
\hline Bleeding (\%) & I (I.7) & $12(13.3)$ & $0.014^{*}$ \\
\hline Death (\%) & $15(25.9)$ & $37(4 I . I)$ & 0.054 \\
\hline
\end{tabular}

Note: *Indicate statistical significance.

Abbreviations: WBC, white blood cells; HGB, hemoglobin; PLT, platelet; ALT, alanine transaminase; AST, aspartate transaminase; TBil, total bilirubin; ALP, alkaline phosphatase; TP, total protein; ALB, albumin; GGT, $\gamma$-glutamyl transpeptidase; BUN, blood urea nitrogen; Cr, creatinine; eGFR, estimated glomerular filtration rate; PT, prothrombin time; Fib, fibrinogen; aPTT, activated partial thromboplastin time; PCT, procalcitonin; CPZ/SAM, cefoperazone/sulbactam.

hypofibrinogenemia group and the normal group were $41.1 \%$ and $25.9 \%$ ( $p=0.054)$ (Table 1). According to the logistic regression analysis, hypofibrinogenemia was independently associated with bleeding (OR 8.96, 95\% CI [1.132-70.946], $p=0.038$ ), but not death (OR 2.009, 95\% CI [0.952-4.24], $p=0.067)$. 
Table 2 Multivariate Analysis of Risk Factors for TigecyclineAssociated Hypofibrinogenemia

\begin{tabular}{|l|l|l|l|}
\hline Variable & Odds Ratio & $\mathbf{9 5 \%} \mathbf{~ C l}$ & P-value \\
\hline Age & 1.037 & $1.002-1.072$ & 0.035 \\
Treatment duration & 1.095 & $1.003-1.196$ & 0.044 \\
Basal fibrinogen & 0.489 & $0.308-0.777$ & 0.002 \\
\hline
\end{tabular}

Abbreviation: $\mathrm{Cl}$, confidence interval.

Table 3 The Sensitivity and Specificity of the Best Cutoff Point of Age, Treatment Duration and the Baseline Fibrinogen Level as a Discriminator of the Probability of Hypofibrinogenemia

\begin{tabular}{|l|l|l|}
\hline Variable & Sensitivity (\%) & Specificity (\%) \\
\hline Age $\geq 82$ years & 0.578 & 0.638 \\
Mediation $\geq 9$ days & 0.789 & 0.414 \\
Basal fibrinogen $\leq 3.5 \mathrm{~g} / \mathrm{L}$ & 0.759 & 0.578 \\
\hline
\end{tabular}

\section{Discussion}

The increase in multidrug-resistant bacteria infections has become a major problem that threatens human health, especially among elderly people. Tigecycline has wide-spectrum antibacterial activity, especially against multidrug-resistant bacteria. It is often used for treating severe infections caused by multidrug-resistant bacteria, sometimes with high doses and extended treatments. But data addressing its safety are limited. In premarketing clinical trials, the most common side effect is digestive-system problems. Adverse reactions of the coagulation system are not common $(<2 \%) .{ }^{8}$ However, according to postmarketing studies, tigecycline is associated with hypofibrinogenemia. To date, most of the published articles about tigecycline-associated hypofibrinogenemia are case reports, ${ }^{8,11,12,16-20}$ and few of them are retrospective observation studies. ${ }^{10,13,14,21}$ Our study was a retrospective study that included 148 patients. We found that patients who were administered tigecycline experienced a prolonged PT, a prolonged APTT, a prolonged TT, and a decreased fibrinogen level. According to our research, hypofibrinogenemia was common in clinical practice, which occurred in $60.8 \%$ of the patients. This is in agreement with prior reports: in Hu's research, ${ }^{14}$ there were $56 \%$ developed hypofibrinogenemia, and in Zhang's research, ${ }^{15}$ there were $50.5 \%$ developed hypofibrinogenemia, respectively. Fibrinogen decreased by an average of $49.2 \%$, PT, aPTT and TT increased by an average of $16.8 \%, 19.5 \%$ and $26.7 \%$, respectively. All of these differences were statistically significant. There was a general decline in fibrinogen. The fibrinogen level decreased from baseline during treatment in $95.9 \%$ of patients, and $60.8 \%$ of patients had hypofibrinogenemia during treatment, which is consistent with the results of previous studies. Cui et al summarized 13 case reports and 4 retrospective observational studies and found that tigecycline can cause coagulation abnormalities, including a prolonged PT, a prolonged APTT and a decreased fibrinogen level after treatment, which recovered after discontinuation. ${ }^{9}$ Leng and $\mathrm{Hu}$ also reached the same conclusion. ${ }^{10,14}$

Hypofibrinogenemia may be acquired or inherited. ${ }^{22}$ In this study, the patients' levels of fibrinogen were within the normal range before the administration of tigecycline. The common causes of acquired hypofibrinogenemia include a) liver disease, as severe liver disease can impair the synthesis function of liver cells, leading to a decrease in fibrinogen. ${ }^{23}$ In this study, liver function did not differ significantly before and after the administration of tigecycline. b) DIC is a well-defined condition of consumptive coagulopathy that can lead to hypofibrinogenemia. ${ }^{24}$ In this study, we exclude the patients who were diagnosed with DIC. Meanwhile, D-dimer is an important factor in the diagnosis of DIC, and there was no significant difference in D-dimer before and after the treatment. c) Other factors, including tumors and drugs, have also been reported to be associated with hypofibrinogenemia. ${ }^{25}$ However, there was no significant difference in tumor incidence between the normal group and the hypofibrinogenemia group in this study. Hypofibrinogenemia appeared after tigecycline was administered and improved when tigecycline was discontinued. Therefore, hypofibrinogenemia was related to tigecycline administration.

According to our study, independent risk factors for tigecycline-associated hypofibrinogenemia included patient age, tigecycline treatment duration, and the baseline fibrinogen level. Consistent with the results of previous studies, the duration of medication and the baseline fibrinogen level were associated with hypofibrinogenemia. ${ }^{14}$ However, the correlation between age and hypofibrinogenemia is controversial. In clinical practice, tigecycline is often used to treat life-threatening infections with multidrug-resistant bacteria, which is often occurred in patients with advanced age. As this study showed, the patients treated with tigecycline were mainly the elderly over 60 years old, most of them had serious infections and with high mortality. Different from previous studies, advanced age was a risk factor for tigecycline-associated hypofibrinogenemia. Zhang et al found that the incidence of hypofibrinogenemia was not related to 
age. ${ }^{13}$ However, their study had a small sample size of 20 patients, and the mean patient age was $62.5 \pm 22.1$ years old, which was $77.09 \pm 15.11$ years old in our study. Similarly, Hu's study also showed there is no correlation between hypofibrinogenemia and age. ${ }^{14}$ But in their study, the age of the hypofibrinogenemia group was $63.72 \pm 15.68$ years old, and that of the normal group was $55.82 \pm 18.59$ years old, which were much smaller than the age of the patients in our study. Our study can better reflect the effect of tigecycline on coagulation in elderly patients. And according to our study, elderly patients are more prone to developing hypofibrinogenemia after the administration of tigecycline.

No correlations were found between the medication dose and infection site. Inconsistent with our findings, $\mathrm{Hu}$ et al found that the dosage of medication and intraabdominal infection were related to the incidence of hypofibrinogenemia. ${ }^{14}$ This inconsistency may be due to differences in the patient characteristics. The study population in Hu's research included mainly patients in the intensive care unit. Although the main infection site was the lung, the proportion of intra-abdominal infections and bloodstream infections was relatively high. The population in our study included both critically ill patients and patients in the general wards. The main infection site was also the lung, but the proportion of patients with intraabdominal infections and bloodstream infections was relatively low. Similarly, in our study, only a small number of patients (15 of 138) were treated with high-dose tigecycline. We further grouped the patients according to the medication dose of tigecycline, compared PT, aPTT, fibrinogen, and TT before and after the application of normaldose and high-dose tigecycline, and found that there was no statistical difference. However, in the high-dose group, after the application of tigecycline, the degree of fibrinogen reduction was greater than the normal-dose group (2.6 $\mathrm{g} / \mathrm{L}$ vs $1.8 \mathrm{~g} / \mathrm{L}, p=0.009$ ), which reached statistical significance. Therefore, according to our research, the application of high-dose tigecycline will also lead to a significant reduction in the concentration of serum fibrinogen. However, due to the limitation of the number of high-dose cases, the incidence of hypofibrinogenemia in the two groups did not reach statistical significance.

We developed risk assessment criteria for tigecyclineassociated hypofibrinogenemia, which can better determine the risk of hypofibrinogenemia after tigecycline administration. Moreover, in our study, hypofibrinogenemia was associated with bleeding. By identifying highrisk patients with hypofibrinogenemia, adjusting treatment programs or closely monitoring patients, we can reduce the occurrence of adverse clinical outcomes. Furthermore, it may be impractical to recommend the routine monitoring of coagulation in all patients using tigecycline, as routine monitoring increases the cost of treatment. Therefore, with these risk assessment criteria, only monitoring coagulation in high-risk patients can reduce the corresponding expenditures. The criteria included an age of $\geq 82$ years, $\geq 9$ days of medication, and a baseline fibrinogen level of $\leq 3.5 \mathrm{~g} / \mathrm{L}$. Patients who meet the criteria are at high risk of hypofibrinogenemia and require close monitoring of coagulation. Plasma infusion and fibrinogen infusion raised the plasma fibrinogen levels in our study. Fibrinogen replacement therapy is effective in the treatment of acute bleeding or the prevention of bleeding complications. ${ }^{26}$ Advanced age is the risk factor for thrombosis and cardiovascular disease. So thrombotic and bleeding events were needed to be carefully considered in elderly patients.

The mechanism of tigecycline-induced hypofibrinogenemia remains controversial. The common mechanism by which antibiotics affect coagulation is that the antibiotics destroy the intestinal flora and inhibit the synthesis of vitamin $\mathrm{K}$, leading to vitamin $\mathrm{K}$-dependent coagulation disorders. However, previous studies have shown that vitamin $\mathrm{K}$ supplementation is not effective for treating hypofibrinogenemia after the administration of tigecycline. ${ }^{9}$ The physiological concentration of fibrinogen in plasma is $1.80-4.20 \mathrm{~g} / \mathrm{L}^{27}$ And the reference ranges of plasma fibrinogen varies from region to region and among different laboratories. It is the highest concentration of proteins among the plasma coagulation factors. Fibrinogen is involved in the coagulation, which plays a central role in the hemostatic and thrombosis process. ${ }^{27}$ The half-life of fibrinogen is $3-4$ days, and it is an acutephase reaction protein. Fibrinogen production is regulated by cytokines. IL- 6 promotes fibrinogen biosynthesis, while IL-1, IL-4, IL-10, IL-13, and tumor necrosis factor- $\alpha$ (TNF- $\alpha$ ) inhibit fibrinogen synthesis. ${ }^{28}$ Tigecycline can reduce the levels of IL- 6 and TNF- $\alpha$, which may interfere with fibrinogen production. ${ }^{29-31}$ At the same time, Brandtner's in vitro studies showed that after adding a supratherapeutic dose of tigecycline to the blood of healthy adults, the changes in fibrinogen levels and coagulation parameters were not clinically significant. However, the addition of supra-therapeutic tigecycline to the cell culture fluid in vitro significantly reduced the 
viability of hepatocytes. ${ }^{32}$ It is speculated that tigecycline may inhibit the liver's synthetic function.

This study has certain limitations. A) This study is a retrospective study. Selection bias may be present and may have affected the results. B) The risk assessment criteria have not been verified externally or included in intervention studies. Therefore, the clinical value of the risk assessment criteria needs to be evaluated in future studies. In the future, it is necessary to verify the clinical value of the risk assessment criteria in large-scale cohort studies. Further randomized controlled trials and animal experiments need to be done to explore the mechanisms of tigecycline-associated hypofibrinogenemia.

\section{Conclusion}

Hypofibrinogenemia is a common adverse effect of tigecycline in our study. It is independently associated with bleeding but not death. Tigecycline-associated hypofibrinogenemia is associated with age, tigecycline treatment duration and baseline fibrinogen level. An age of $\geq 82$ years, $\geq 9$ days of medication, and a fibrinogen level of $\leq 3.5 \mathrm{~g} / \mathrm{L}$ are risk factors. Elderly patients are more prone to developing hypofibrinogenemia after the administration of tigecycline. With this risk assessment criteria, clinicians can selectively monitor coagulation to reduce the corresponding expenditures.

\section{Ethics Approval and Informed Consent}

The Peking University Third Hospital Medical Science Research Ethics Committee approved the study. The procedures used in this study adhere to the tenets of the Declaration of Helsinki. Considering the characteristics of the study (observational retrospective cohort study), the ethical committee allowed us the exemption of obtaining informed consent.

\section{Funding}

The study was not funded.

\section{Disclosure}

The authors report no conflicts of interest in this work.

\section{References}

1. Hylands J. Tigecycline: a new antibiotic. Intensive Crit Care Nurs. 2008;24(4):260-263. doi:10.1016/j.iccn.2008.03.006
2. Rose WE, Rybak MJ. Tigecycline: first of a new class of antimicrobial agents. Pharmacotherapy. 2006;26(8):1099-1110. doi:10.1592/ phco.26.8.1099

3. Brink AJ, Bizos D, Boffard KD, et al. Guideline: appropriate use of tigecycline. S Afr Med J. 2010;100(6 Pt 2):388-394. doi:10.7196/ SAMJ.4109

4. Rubinstein E, Vaughan D. Tigecycline: a novel glycylcycline. Drugs. 2005;65(10):1317-1336. doi:10.2165/00003495-20056510 0-00002

5. Kurihara MNL, Sales ROD, Silva KED, Maciel WG, Simionatto S. Multidrug-resistant Acinetobacter baumannii outbreaks: a global problem in. Rev Soc Bras Med Tro. 2020;53:e20200248. doi:10.1590/ 0037-8682-0248-2020

6. Xia G, Jiang R. Clinical study on the safety and efficacy of high-dose tigecycline in the elderly. Medicine. 2020;99(10):e19466. doi:10. 1097/MD.0000000000019466

7. Tasina E, Haidich AB, Kokkali S, Arvanitidou M. Efficacy and safety of tigecycline for the treatment of infectious diseases: a meta-analysis. Lancet Infect Dis. 2011;11(11):834-844. doi:10.1016/ S1473-3099(11)70177-3

8. McMahan J, Moenster RP. Tigecycline-induced coagulopathy. Am $J$ Health Syst Pharm. 2017;74(3):130-134. doi:10.2146/ajhp150894

9. Cui N, Cai H, Li Z, Lu Y, Wang G, Lu A. Tigecycline-induced coagulopathy: a literature review. Int J Clin Pharm. 2019;41 (6):1408-1413. doi:10.1007/s11096-019-00912-5

10. Leng B, Xue YC, Zhang W, Gao TT, Yan GQ, Tang H. A retrospective analysis of the effect of tigecycline on coagulation function. Chem Pharm Bull (Tokyo). 2019;67(3):258-264. doi:10. 1248/cpb.c18-00844

11. Giryes S, Azzam ZS, Ismael-Badarneh R, Krivoy N, Berger G. Severe coagulation disorder and thrombocytopenia associated with tigecycline - case report and review of literature. Curr Drug Saf. 2017;12(1):7-9. doi:10.2174/1574886311666160920090714

12. Wu X, Zhao P, Dong L, Zhang X. A case report of patient with severe acute cholangitis with tigecycline treatment causing coagulopathy and hypofibrinogenemia. Medicine (Baltimore). 2017;96(49):e9124. doi:10.1097/MD.0000000000009124

13. Zhang Q, Zhou S, Zhou J. Tigecycline treatment causes a decrease in fibrinogen levels. Antimicrob Agents Ch. 2015;59(3):1650-1655. doi:10.1128/AAC.04305-14

14. Hu J, Xiao Y, Zheng Y, Lai Y, Fang X, Fang Q. Clinical characteristics and risk factors of tigecycline-associated hypofibrinogenaemia in critically ill patients. Eur J Clin Pharmacol. 2020;76(7):913-922. doi:10.1007/s00228-020-02860-w

15. Zhang Q, Wang J, Liu H, Ma W, Zhou S, Zhou J. Risk factors for tigecycline-induced hypofibrinogenaemia. J Clin Pharm Ther. 2020; 45(6):1434-1441. doi:10.1111/jcpt.13250

16. Wu PC, Wu CC. Tigecycline-associated hypofibrinogenemia: a case report and review of the literature. IDCases. 2018;11:56-57. doi:10. 1016/j.idcr.2018.01.003

17. Yilmaz DF, Yildirim H, Sen EM. A lesser known side effect of tigecycline: hypofibrinogenemia. Turk J Haematol. 2018;35(1): 83-84. doi:10.4274/tjh.2017.0310

18. Rossitto G, Piano S, Rosi S, Simioni P, Angeli P. Life-threatening coagulopathy and hypofibrinogenaemia induced by tigecycline in a patient with advanced liver cirrhosis. Eur $J$ Gastroenterol Hepatol. 2014;26(6):681-684. doi:10.1097/MEG.0000000000000 087

19. Pieringer H, Schmekal B, Biesenbach G, Pohanka E. Severe coagulation disorder with hypofibrinogenemia associated with the use of tigecycline. Ann Hematol. 2010;89(10):1063-1064. doi:10.1007/ s00277-010-0911-7

20. Sabanis N, Paschou E, Gavriilaki E, Kalaitzoglou A, Vasileiou S. Hypofibrinogenemia induced by tigecycline: a potentially life-threatening coagulation disorder. Infect Dis (Lond). 2015;47 (10):743-746. doi:10.3109/23744235.2015.1043942 
21. Campany-Herrero D, Larrosa-Garcia M, Lalueza-Broto $P$, et al. Tigecycline-associated hypofibrinogenemia in a real-world setting. Int J Clin Pharm-Net. 2020;42(4):1184-1189. doi:10.1007/s11096-02001072-7

22. Simurda T, Zolkova J, Snahnicanova Z, et al. Identification of two novel fibrinogen Bbeta chain mutations in two Slovak families with quantitative fibrinogen disorders. Int J Mol Sci. 2017;19(1):100. doi:10.3390/ijms 19010100

23. Casini A, Undas A, Palla R, Thachil J, de Moerloose P. Diagnosis and classification of congenital fibrinogen disorders: communication from the SSC of the ISTH. J Thromb Haemost. 2018;16 (9):1887-1890. doi:10.1111/jth.14216

24. Toh CH, Alhamdi Y, Abrams ST. Current pathological and laboratory considerations in the diagnosis of disseminated intravascular coagulation. Ann Lab Med. 2016;36(6):505-512. doi:10.3343/ alm.2016.36.6.505

25. Nair RR, Chauhan R, Harankhedkar S, Mahapatra M, Saxena R. Imatinib-induced platelet dysfunction and hypofibrinogenemia in chronic myeloid leukemia. Blood Coagulation Fibrinolysis. 2019;30(5):246-248. doi:10.1097/MBC.0000000000000817

26. Simurda T, Vilar R, Zolkova J, et al. A novel nonsense mutation in FGB (c.1421G $>$ A; p.Trp474Ter) in the beta chain of fibrinogen causing hypofibrinogenemia with bleeding phenotype. Biomedicines. 2020;8(12):605. doi:10.3390/biomedicines81 20605
27. Simurda T, Brunclikova M, Asselta R, et al. Genetic variants in the FGB and FGG genes mapping in the beta and gamma nodules of the fibrinogen molecule in congenital quantitative fibrinogen disorders associated with a thrombotic phenotype. Int J Mol Sci. 2020;21 (13):4616. doi:10.3390/ijms21134616

28. Green F, Humphries S. Control of plasma fibrinogen levels. Baillieres Clin Haematol. 1989;2(4):945-959. doi:10.1016/S0950-3536(89)80053-8

29. Saliba R, Paasch L, El SA. Tigecycline attenuates staphylococcal superantigen-induced T-cell proliferation and production of cytokines and chemokines. Immunopharmacol Immunotoxicol. 2009;31(4): 583-588. doi:10.3109/08923970902838672

30. Pichereau S, Moran JJ, Hayney MS, Shukla SK, Sakoulas G, Rose WE. Concentration-dependent effects of antimicrobials on Staphylococcus aureus toxin-mediated cytokine production from peripheral blood mononuclear cells. J Antimicrob Chemother. 2012;67 (1):123-129. doi:10.1093/jac/dkr417

31. Salvatore CM, Techasaensiri C, Tagliabue C, et al. Tigecycline therapy significantly reduces the concentrations of inflammatory pulmonary cytokines and chemokines in a murine model of Mycoplasma pneumoniae pneumonia. Antimicrob Agents Chemother. 2009;53 (4):1546-1551. doi:10.1128/AAC.00979-08

32. Brandtner A, Bachler M, Fries D, et al. Tigecycline interferes with fibrinogen polymerization independent of peripheral interactions with the coagulation system. Antibiotics (Basel). 2020;9(2):84. doi:10. 3390/antibiotics 9020084

\section{Publish your work in this journal}

Therapeutics and Clinical Risk Management is an international, peerreviewed journal of clinical therapeutics and risk management, focusing on concise rapid reporting of clinical studies in all therapeutic areas, outcomes, safety, and programs for the effective, safe, and sustained use of medicines. This journal is indexed on PubMed Central, CAS,
EMBase, Scopus and the Elsevier Bibliographic databases. The manuscript management system is completely online and includes a very quick and fair peer-review system, which is all easy to use. Visit http://www.dovepress.com/testimonials.php to read real quotes from published authors 